Check for updates

Cite this: Phys. Chem. Chem. Phys., 2018, 20, 23441

Received 25th June 2018, Accepted 14th August 2018

DOI: $10.1039 / c 8 c p 04020 f$

rsc.li/pccp

\section{Rotationally resolved electronic spectroscopy of 3-cyanoindole and the 3-cyanoindole-water complex $\dagger$}

\author{
Michael Schneider, (D) a Marie-Luise Hebestreit, (D) ${ }^{a}$ Mirko Matthias Lindic, (D) ${ }^{a}$ \\ Hilda Parsian, ${ }^{a}$ América Yareth Torres-Boy, ${ }^{b}$ Leonardo Álvarez-Valtierra, (DD ${ }^{b}$ \\ W. Leo Meerts, $\mathbb{D}^{c}$ Ralf Kühnemuth ${ }^{d}$ and Michael Schmitt (D) *a
}

\begin{abstract}
The rotationally resolved electronic spectra of the origin bands of 3-cyanoindole, 3-cyanoindole $\left(\mathrm{d}_{1}\right)$, and the 3-cyanoindole- $\left(\mathrm{H}_{2} \mathrm{O}\right)_{1}$ cluster have been measured and analyzed using evolutionary algorithms. For the monomer, permanent dipole moments of $5.90 \mathrm{D}$ for the ground state, and of $5.35 \mathrm{D}$ for the lowest excited singlet state have been obtained from electronic Stark spectroscopy. The orientation of the transition dipole moment is that of $a{ }^{1}{ }^{1} \mathrm{~L}_{\mathrm{b}}$ state for the monomer. The water moiety in the water cluster could be determined to be trans-linearly bound to the $\mathrm{NH}$ group of 3-cyanoindole, with an $\mathrm{NH} \ldots \mathrm{O}$ hydrogen bond length of $201.9 \mathrm{pm}$ in the electronic ground state. Like the 3-cyanoindole monomer, the 3 -cyanoindole-water cluster also shows an ${ }^{1} \mathrm{~L}_{b}$-like excited singlet state. The excited state lifetime of isolate 3 -cyanoindole in the gas phase has been determined to be $9.8 \mathrm{~ns}$, and that of 3-cyanoindole $\left(\mathrm{d}_{1}\right)$ has been found to be $14.8 \mathrm{~ns}$, while that of the $1: 1$ water cluster is considerably shorter (3.6 ns). The excited state lifetime of 3 -cyanoindole $\left(d_{1}\right)$ in $D_{2} O$ solution has been found to be smaller than 20 ps.
\end{abstract}

\section{Introduction}

Understanding solvent reorganization processes at a molecular level is key to the determination of dipole moments of electronically excited molecules in solution. Beforehand, the driving forces which determine the solvent orientation in both electronic states have to be investigated on a molecular level. Indole derivatives are especially interesting in this respect, since they have two energetically close-lying excited singlet states, which have considerably different dipole moments. Dipole-dipole interactions with polar solvent molecules lead to different stabilization of the electronically excited states. Depending on the dipole moments, even the energetic order of the lowest excited singlet states might switch upon cluster formation or solvation in polar solvents. These states are called ${ }^{1} \mathrm{~L}_{\mathrm{a}}$ and ${ }^{1} \mathrm{~L}_{\mathrm{b}}$ in the nomenclature of Platt, ${ }^{1}$ adapted to indole by Weber. ${ }^{2}$

\footnotetext{
${ }^{a}$ Heinrich-Heine-Universität, Institut für Physikalische Chemie I, D-40225 Düsseldorf, Germany. E-mail: mschmitt@uni-duesseldorf.de; Fax: +49 211 8113689; Tel: +49 2118112100

${ }^{b}$ División de Ciencias e Ingenierías, Universidad de Guanajuato-Campus León, León, Guanajuato 37150, Mexico

${ }^{c}$ Radboud University, Institute for Molecules and Materials, Felix Laboratory, Toernooiveld 7c, 6525 ED Nijmegen, The Netherlands

${ }^{d}$ Heinrich-Heine-Universität, Lehrstuhl für Molekulare Physikalische Chemie, D-40225 Düsseldorf, Germany

$\dagger$ Electronic supplementary information (ESI) available. See DOI: 10.1039/c8cp04020f
}

The ${ }^{1} \mathrm{~L}_{\mathrm{b}}$ state has a small dipole moment and is lower in energy in apolar surroundings, while the ${ }^{1} \mathrm{~L}_{\mathrm{a}}$ state on the other hand has a comparatively large dipole moment, leading to an inversion of the electronic state ordering in polar solvents. Some molecular systems change the energetic order of the excited states already upon addition of the first water molecules to the chromophore like azaindole. ${ }^{3}$

Indole, as the chromophore of the aromatic amino acid tryptophane, has been extensively studied, mainly regarding the location of the higher electronically excited ${ }^{1} \mathrm{~L}_{\mathrm{a}}$ state relative to the lowest ${ }^{1} \mathrm{~L}_{\mathrm{b}}$ state, both experimentally ${ }^{4-12}$ as well as theoretically. ${ }^{13-18}$

Exchange of one hydrogen atom in the chromophore for the highly polar cyano group, changes the fluorescence properties of the indole chromophore considerably. ${ }^{19,20} 5$-Cyanotryptophane is used as a fluorescence probe of protein hydration. ${ }^{21}$ Its chromophore, 5-cyanoindole, has been studied using rotationally resolved electronic spectroscopy and fluorescence emission spectroscopy in our group. ${ }^{22-24}$ For 5-cyanoindole, we found that the lowest electronically excited singlet state is of ${ }^{1} \mathrm{~L}_{\mathrm{a}}$ character. The question arises, if for other substitution positions, the cyano group has the same stabilizing effect on the ${ }^{1} \mathrm{~L}_{\mathrm{a}}$ state. Therefore, we varied the position from the 5-position, which is located in the benzene ring of the chromophore, to the 3-position within the pyrrolic ring, in the present study. The 3-cyanoindole system is of general interest, since differently cyano-substituted indole derivatives find increasing interest as small fluorescence reporters in fluorescent proteins. ${ }^{19,20}$ 
Considerably less work has been performed on hydrogen bonded clusters of indole or indole derivatives. Korter et al. ${ }^{25}$ could show that the binary indole-water complex undergoes considerable solvent reorientation upon excitation to the lowest excited singlet state. The lowest excited singlet states of both the monomer and the water cluster, in which the water is bound linearly to the pyrrolic $\mathrm{NH}$ group, have been shown to be of ${ }^{1} \mathrm{~L}_{\mathrm{b}}$ character. The dipole moment of the indole-water complex was determined by Kang and Pratt using rotationally resolved electronic Stark spectroscopy. ${ }^{9}$ They found that the dipole of the water molecule induces a dipole moment in the indole moiety, which amounts to a substantial fraction of $30-40 \%$ of the total dipole moment of the cluster. Azaindolewater clusters have been examined using high resolution laser induced fluorescence (HRLIF) spectroscopy. It could be shown that the lowest excited singlet state has ${ }^{1} \mathrm{~L}_{\mathrm{b}}$ character, while the excitation in the $n=1$ and $n=2$ water clusters is to the ${ }^{1} \mathrm{~L}_{\mathrm{a}}$ state. ${ }^{3}$

Ahn et al. ${ }^{26}$ investigated 3-cyanoindole and several 3-cyanoindolewater clusters, using mass-selected resonant two-photon ionization (R2PI) and UV-UV hole-burning (UVHB) spectroscopy. Assignments of the different cluster structures were made on the basis of a Franck-Condon analysis of the vibronic spectra. From the comparison of experiment and FC simulation they concluded that only the most stable conformer is present in the molecular beam. According to ab initio calculations, this is the linear $\mathrm{N}-\mathrm{H} \cdots \mathrm{O}$ hydrogen bond complex.

In the present study, we investigate the electronic nature of the lowest excited state of 3-cyanoindole, its dipole moments in both ground and excited singlet states and the geometry and electronic nature of the binary 3-cyanoindole-water cluster using a combination of rotationally resolved fluorescence spectroscopy, rotationally resolved electronic Stark spectroscopy, time-correlated single photon counting, and ab initio quantum chemical calculations for a deeper understanding of the photophysics of electronically excited 3-cyanoindole.

\section{Experimental section}

\subsection{Experimental procedures}

2.1.1 Rotationally resolved electronic (Stark) spectroscopy. 3-Cyanoindole (95\%) was purchased from Oxchem and used without further purification. To record rotationally resolved electronic spectra, the sample was heated to $200{ }^{\circ} \mathrm{C}$ and co-expanded with 300 mbar of argon into the vacuum through a $200 \mu \mathrm{m}$ nozzle. After the expansion, a molecular beam was formed using two skimmers $(1 \mathrm{~mm}$ and $3 \mathrm{~mm}$ ) linearly aligned inside a differentially pumped vacuum system consisting of three vacuum chambers. The molecular beam was crossed at right angles with the laser beam $360 \mathrm{~mm}$ downstream of the nozzle. To create the excitation beam, $10 \mathrm{~W}$ of the $532 \mathrm{~nm}$ line of a diode pumped solid state laser (Spectra-Physics Millennia eV) pumped a single frequency ring dye laser (Sirah Matisse DS) operated with Rhodamine 110. The fluorescence light of the dye laser was frequency doubled in an external folded ring cavity (Spectra Physics Wavetrain) with a resulting power of about 10-15 $\mathrm{mW}$ during the experiments. The fluorescence light of the samples was collected perpendicular to the plane defined by the laser and molecular beam by an imaging optics setup consisting of a concave mirror and two planoconvex lenses onto the photocathode of a UV enhanced photomultiplier tube (Thorn EMI 9863QB). The signal output was then discriminated and digitized by a photon counter and transmitted to a PC for data recording and processing. The relative frequency was determined using a quasi confocal Fabry-Perot interferometer. The absolute frequency was obtained by comparing the recorded spectrum to the tabulated lines in the iodine absorption spectrum. ${ }^{27}$ A detailed description of the experimental setup for rotationally resolved laser induced fluorescence spectroscopy has been given previously. ${ }^{28,29}$ The Stark plates consist of a parallel pair of electroformed nickel wire grids (18 mesh per mm, $50 \mathrm{~mm}$ diameter) with a transmission of $95 \%$ in the UV. Their effective distance is $23.49 \pm 0.05 \mathrm{~mm}$, symmetrically aligned with respect to the laser beam. This distance was calibrated using the accurately known dipole moment of benzonitrile. ${ }^{30,31}$ The polarization plane of the incoming laser beam can be rotated by $90^{\circ}$ inside the vacuum chamber by means of an achromatic $\lambda / 2$ plate (Bernhard Halle $240-380 \mathrm{~nm}$ ). The $\lambda / 2$ plate can be pushed in or pulled out of the laser beam using a linear motion vacuum feedthrough.

2.1.2 Time-correlated single photon counting. Time-correlatedsingle-photon-counting (TCSPC) was performed with a DeltaFlex Ultima spectrometer (HORIBA Jobin Yvon), equipped with a supercontinuum light source SuperK Extreme EXR-20 and frequency doubler SuperK Extend-UV/DUV (both NKT Photonics). The signal was recorded under magic angle conditions, a pulse repetition rate of $19.2 \mathrm{MHz}$ and a sample temperature of $20^{\circ}$. The instrument response function (IRF, FWHM approximately $60 \mathrm{ps}$ ) was recorded using a scattering solution (Ludox) for each excitation wavelength and used in the fits applying an iterative reconvolution algorithm to minimize $\chi^{2}$.

\subsection{Quantum chemical calculations}

Structure optimizations were performed employing Dunning's correlation-consistent polarized valence triple zeta (cc-pVTZ) basis set from the Turbomole library. ${ }^{32,33}$ The equilibrium geometries of the electronic ground and the lowest excited singlet states were optimized using the approximate coupled cluster singles and doubles model (CC2) employing the resolution-of-the-identity approximation (RI). ${ }^{34-36}$ For the structure optimizations spincomponent scaling (SCS) modifications to CC2 were taken into account. ${ }^{37}$ Vibrational frequencies and zero-point corrections to the adiabatic excitation energies were obtained from numerical second derivatives using the NumForce script. ${ }^{38}$ For the cluster stabilization energies, the basis set superposition errors (BSSE) have been accounted for, using the counterpoise corrections described by Boys and Bernardi ${ }^{39}$ and implemented in the JOBBSSE jobscript of turbomole. Additionally, the equilibrium geometries of the lowest electronically excited singlet states were geometry optimized by means of a time-dependent density functional theory (TD-DFT) gradient ${ }^{40,41}$ using the Becke, threeparameter, Lee-Yang-Parr (B3-LYP) density functional. ${ }^{42}$ 


\subsection{Fits of the rovibronic spectra using evolutionary algorithms}

Evolutionary algorithms allow us to make a quick and successful automatic assignment of the rotationally resolved spectra, even for large molecules and dense spectra. ${ }^{43-46}$ Beside a correct Hamiltonian to describe the spectrum and reliable intensities inside the spectrum, an appropriate search method is needed. Evolutionary strategies are a powerful tool to handle complex multi-parameter optimizations and find the global optimum. For the analysis of the presented high-resolution spectra, we used the covariance matrix adaptation evolution strategy (CMA-ES), which is described in detail elsewhere. ${ }^{47,48}$ In this variant of global optimizers mutations are adapted via a CMA mechanism to find the global minimum, even on rugged search landscapes that are additionally complicated due to noise, local minima and/or sharp bends.

\section{Results}

\subsection{Computational results}

3.1.1 3-Cyanoindole. The optimization of 3-cyanoindole in the ground and the lowest excited singlet state yields a planar structure in both states both with genuine CC2 as well as with SCS-CC2. Contrary to 5-cyanoindole, CC2 and the spin component scaled variant SCS-CC2 obtain the same state as the lowest excited singlet state. ${ }^{22}$ The molecular parameters (rotational constants $A, B$, and $C$ in both electronic states, the inertial defects $\Delta I$, the angle $\theta$ of the transition dipole moment with the inertial $a$ axis, and the zero-point corrected origin frequency $\nu_{0}$ ) are compiled in Table 1 and are compared to the experimental results, which are described in Section 4.1.

The inertial defects of 3-cyanoindole in both states are exactly zero for the equilibrium structures which immediately shows the planarity of the molecule.

The calculated angle of the transition dipole moment with the inertial $a$-axis is $+4^{\circ}, c f$. Fig. 1 . To compare this value better with the transition dipole moment vector of bare indole, the transition dipole moment (TDM) is rotated into the inertial axis frame of indole. This yields an angle of $+52^{\circ}$ with the inertial $a$-axis of indole. The respective values in indole $\left(\mathrm{L}_{\mathrm{b}}\right)$ and 5 -cyanoindole $\left(\mathrm{L}_{\mathrm{a}}\right)$ are $+38^{\circ}$ and $-12^{\circ}$, respectively. Thus, the orientation of the TDM in 3-cyanoindole, is clearly that of an ${ }^{1} \mathrm{~L}_{\mathrm{b}}$ state. The main contributions to the excitation to the lowest excited singlet state are 0.81(LUMO $\leftarrow$ HOMO-1) + 0.43(LUMO $+1 \leftarrow \mathrm{HOMO})+0.24(\mathrm{LUMO}+2 \leftarrow \mathrm{HOMO})$, cf. Fig. 3, which are also representative for a transition to a ${ }^{1} \mathrm{~L}_{\mathrm{b}}$ state. The zero-point corrected adiabatic excitation energy amounts to $36156 \mathrm{~cm}^{-1}\left(36045 \mathrm{~cm}^{-1}\right)$ at the CC2 (SCS-CC2) level of theory. The permanent dipole moment in the ground state amounts to $5.95 \mathrm{D}$ and to $5.51 \mathrm{D}$ in the electronically excited ${ }^{1} \mathrm{~L}_{\mathrm{b}}$ state.

An explicit caveat has to be given for the ${ }^{1} \mathrm{~L}_{\mathrm{a}}$ state optimization. This state is vertically the second excited singlet state at the SCSCC2 level of theory. Optimization of this state leads, independently of the starting geometry, into a conical intersection with the lowest excited state. However, it is well-known, that the order of
Table 1 SCS-CC2/cc-pVTZ computed and experimental molecular parameters of 3 -cyanoindole $(3-\mathrm{Cl})$ and the $\mathrm{N}$-deuterated isotopologue $\left(\left(\mathrm{d}_{1}\right)\right)$. Doubly primed parameters belong to the electronic ground and single primed to the excited state. $\theta_{\mathrm{D}}$ is the angle of the permanent dipole moment vector with the main inertial a-axis. A negative sign of this angle means an anticlockwise rotation of the main inertial a-axis onto the dipole moment vector, shown in Fig. 1. $\theta$ is the angle of the transition dipole moment vector with the main inertial a-axis. The same convention for its sign is used as for $\theta_{\mathrm{D}}$. For details see text

\begin{tabular}{|c|c|c|c|c|}
\hline & \multicolumn{2}{|c|}{ Theory SCS-CC2 } & \multicolumn{2}{|l|}{ Experiment } \\
\hline & 3-CI & $3-\mathrm{CI}\left(\mathrm{d}_{1}\right)$ & 3-CI & 3-CI $\left(\mathrm{d}_{1}\right)$ \\
\hline$A^{\prime \prime} / \mathrm{MHz}$ & 2255 & 2171 & 2258.53(48) & 2180.09(17) \\
\hline$B^{\prime \prime} / \mathrm{MHz}$ & 943 & 942 & $947.38(3)$ & $950.80(2)$ \\
\hline$C^{\prime \prime} / \mathrm{MHz}$ & 665 & 657 & $667.44(3)$ & $660.88(1)$ \\
\hline$\Delta I^{\prime \prime} / \mathrm{amu} \AA^{2}$ & 0 & 0 & -0.02 & -0.02 \\
\hline$\mu_{\mathrm{a}}^{\prime \prime} / \mathrm{D}$ & +3.94 & +3.96 & $\pm 4.15(1)$ & - \\
\hline$\mu_{\mathrm{b}}^{\prime \prime} / \mathrm{D}$ & -4.38 & -4.34 & $\pm 4.20(1)$ & - \\
\hline$\mu^{\prime \prime} / \mathrm{D}$ & 5.89 & 5.89 & $5.90(1)$ & - \\
\hline$\theta_{\mathrm{D}}^{\prime \prime} 1^{\circ}$ & +48.0 & +47.53 & \pm 45.3 & - \\
\hline$A^{\prime} / \mathrm{MHz}$ & 2221 & 2140 & $2227.15(49)$ & $2150.84(18)$ \\
\hline$B^{\prime} / \mathrm{MHz}$ & 931 & 931 & $937.46(4)$ & $938.81(3)$ \\
\hline$C^{\prime} / \mathrm{MHz}$ & 656 & 649 & $659.83(4)$ & $653.59(2)$ \\
\hline$\Delta I^{\prime} / \mathrm{amu} \AA^{2}$ & 0 & 0 & -0.08 & -0.06 \\
\hline$\mu_{\mathrm{a}}^{\prime} / \mathrm{D}$ & +3.71 & +3.75 & $\pm 4.14(1)$ & - \\
\hline$\mu_{\mathrm{b}}^{\prime} / \mathrm{D}$ & -3.91 & -3.87 & $\pm 3.39(1)$ & - \\
\hline$\mu^{\prime} / \mathrm{D}$ & 5.39 & 5.39 & $5.35(1)$ & - \\
\hline$\theta_{\mathbf{D}}^{\prime} /^{\circ}$ & +46.5 & +45.92 & \pm 39.3 & - \\
\hline$\Delta A / \mathrm{MHz}$ & -34 & -31 & $-31.38(1)$ & $-27.90(1)$ \\
\hline$\Delta B / \mathrm{MHz}$ & -12 & -12 & $-9.93(1)$ & $-9.64(1)$ \\
\hline$\Delta C / \mathrm{MHz}$ & -9 & -9 & $-7.61(1)$ & $-7.71(1)$ \\
\hline$\Delta \nu_{\text {Lorentz }} / \mathrm{MHz}$ & - & - & $16.31(1)$ & $10.72(1)$ \\
\hline$\tau / \mathrm{ns}$ & - & - & $9.8(1)$ & $14.8(1)$ \\
\hline$\theta /^{\circ}$ & +4 & +4 & $\pm 15.3(1)$ & $\pm 3.16(73)$ \\
\hline$\theta_{\mathrm{T}}{ }^{\circ}$ & -0.45 & -0.45 & $00.52(5)$ & - \\
\hline$\nu_{0} / \mathrm{cm}^{-1}$ & 36045 & 36156 & $35299.36(12)$ & $35306.58(15)$ \\
\hline
\end{tabular}

${ }^{1} \mathrm{~L}_{\mathrm{a}}$ and ${ }^{1} \mathrm{~L}_{\mathrm{b}}$ states is reversed for time-dependent density functional theory (TD-DFT). ${ }^{4-52}$ Therefore, we changed the level of theory to TD-DFT using the B3-LYP functional and the cc-pVTZ basis set. As expected, at the TD-DFT level, the vertically lowest state is the ${ }^{1} \mathrm{~L}_{\mathrm{a}}$ state, well below ${ }^{1} \mathrm{~L}_{\mathrm{b}}$. Geometry optimization led to the lowest excited state, which remained the ${ }^{1} \mathrm{~L}_{\mathrm{a}}$ state. Thus, ${ }^{1} \mathrm{~L}_{\mathrm{a}}$ is also adiabatically the lowest excited singlet state at the TD-DFT level. The fact that TD-DFT employing the B3-LYP functional reverses the order of ${ }^{1} \mathrm{~L}_{\mathrm{a}}$ and ${ }^{1} \mathrm{~L}_{\mathrm{b}}$ states has been observed before in tryptamine. ${ }^{52}$

For tryptamine, the correct energetic order of the excited states could be obtained using density functional multi-reference configuration interaction (DFT-MRCI), which correctly takes dynamic and static electron correlation into account, at the TD-DFT optimized geometries. For 3-cyanoindole, the SCS-CC2 energy of the ${ }^{1} \mathrm{~L}_{\mathrm{a}}$ state, which constitutes the second electronically excited singlet state, was calculated at the geometry of the ${ }^{1} \mathrm{~L}_{\mathrm{b}}$ state at the TD-DFT level, where it is the lowest electronically excited singlet state. In this geometry, ${ }^{1} \mathrm{~L}_{\mathrm{a}}$ is adiabatically also at the SCS-CC2 level the lowest excited singlet state. These findings are summarized in Fig. 2. However, further optimization starting from this geometry again led to the conical intersection with the ${ }^{1} \mathrm{~L}_{\mathrm{b}}$ state.

Using the SCS-CC2 energy at the TD-B3-LYP optimized structure for the ${ }^{1} \mathrm{~L}_{\mathrm{a}}$ state an angle of the TDM with the inertial $a$ axis of $-80^{\circ}$ is calculated. The leading contributions to the 

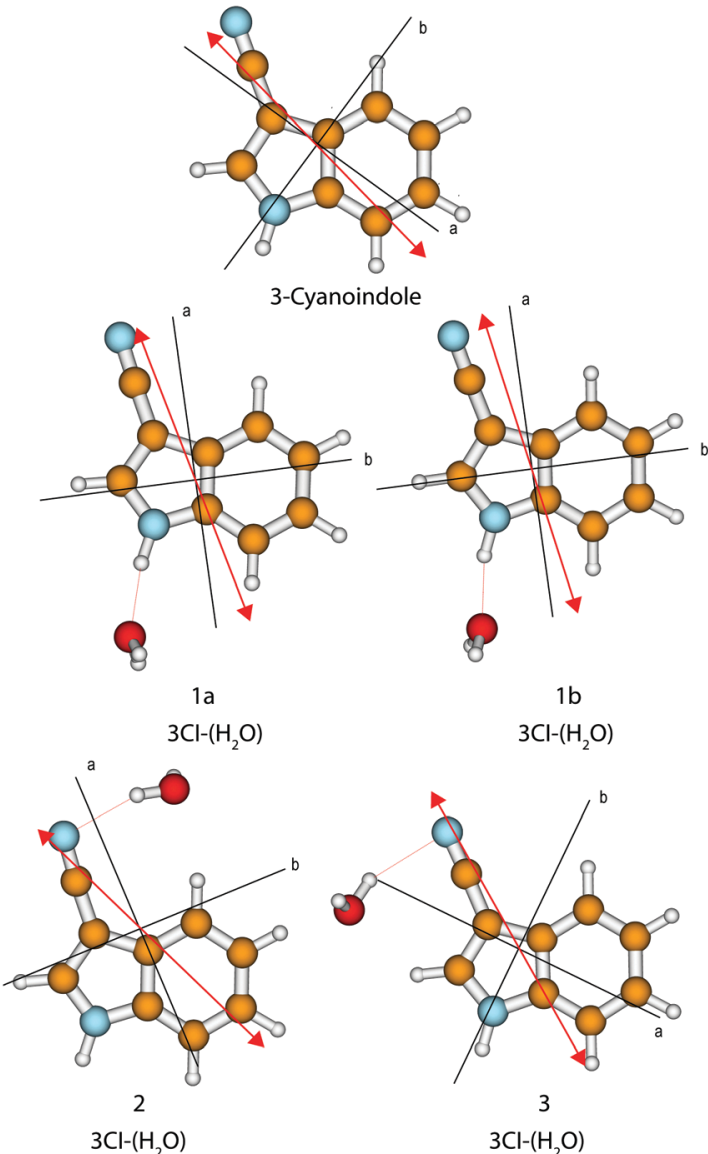

$1 \mathrm{~b}$ $3 \mathrm{Cl}-\left(\mathrm{H}_{2} \mathrm{O}\right)$

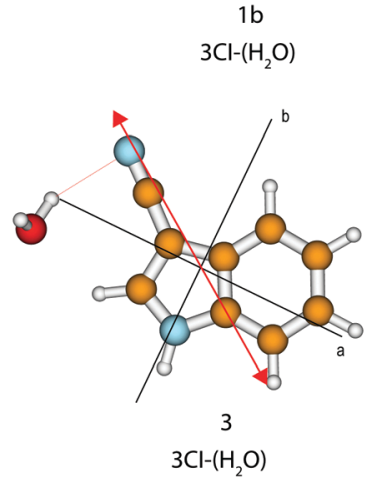

Fig. 1 Calculated structures, inertial axes and transition dipole moments of 3-cyanoindole and the 3-cyanoindole water cluster.

excitation are: $0.87($ LUMO $\leftarrow$ HOMO $)+0.39$ (LUMO-1 $\leftarrow$ $\mathrm{HOMO}+1)$. The zero-point corrected adiabatic excitation energy amounts to $39392 \mathrm{~cm}^{-1}$ and the permanent dipole moment to $5.18 \mathrm{D}$ in the electronically excited ${ }^{1} \mathrm{~L}_{\mathrm{a}}$ state, with an angle of $+31^{\circ}$ to the inertial $a$-axis.

Apart from the two lowest excited $\pi \pi^{*}$ states, the photophysics of indoles is governed by the repulsive $\pi \sigma^{*}$ state (Table 2). We calculated the vertical excitation to all three states at the SCS-CC2/cc-pVTZ level of theory. While the two lowest $\pi \pi^{*}$ states are relatively close at the ground state geometry, there is a larger gap to the $\pi \sigma^{*}$ state, $c f$. Table 2 . The coefficients for this transition are shown in Fig. 3. The $\sigma^{*}$ orbital at the hydrogen atom of the $\mathrm{NH}$ is diffuse and collapses into the hydrogen 1s orbital upon distortion along the $\mathrm{NH}$ coordinate, as has been shown by Sobolewski and Domcke. ${ }^{17,53-55}$ For 3-cyanoindole, the $\pi \sigma^{*}-\pi \pi^{*}$ difference is by more than $1000 \mathrm{~cm}^{-1}$ smaller compared to indole. The dipole moment of the $\pi \sigma^{*}$ state in indole is more than $5 \mathrm{D}$ higher than in the ground state, while for 3-cyanoindole only an increase of $2 \mathrm{D}$ is found. The oscillator strengths of the respective states of indole and 3-cyanoindole are of comparable size, with the ${ }^{1} \mathrm{~L}_{\mathrm{a}}$ oscillator strength between 2 and 3 times larger than for the ${ }^{1} \mathrm{~L}_{\mathrm{b}}$ state.

3.1.2 3-Cyanoindole-water. Several starting geometries for the optimization of the 3-cyanoindole-water cluster have
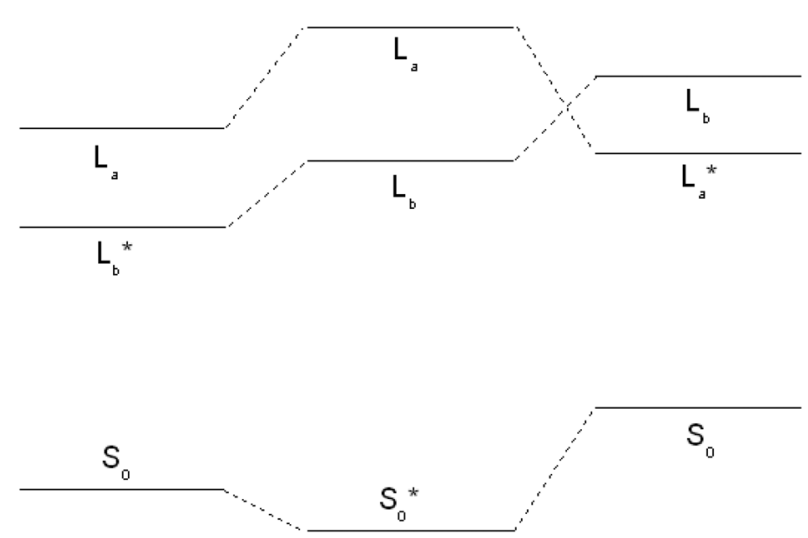

Fig. 2 Energies of the ground and excited ${ }^{1} L_{a}$ and ${ }^{1} L_{b}$ states of 3-cyanoindole from SCS-CC2/cc-pVTZ calculations at the TD-B3-LYP/cc-pVTZ optimized geometries. The optimized state is marked by an asterisk. In the left part of the figure, ${ }^{1} L_{b}$ is the geometry optimized state, in the middle the ground state, and in the right part, the ${ }^{1} L_{a}$ state.

Table 2 SCS-CC2/cc-pVTZ computed vertical excitation energies, relative to the energy of the lowest excitation in $\mathrm{cm}^{-1}$, excited state dipole moment $\mu$ and oscillator strength $f$ of indole and 3-cyanoindole

\begin{tabular}{lcllllll}
\hline & \multicolumn{3}{c}{ Indole } & & & \multicolumn{3}{l}{ 3-Cyanoindole } \\
\cline { 2 - 4 } & $E\left(\mathrm{~cm}^{-1}\right)$ & $\mu(\mathrm{D})$ & $f$ & & $E\left(\mathrm{~cm}^{-1}\right)$ & $\mu(\mathrm{D})$ & $f$ \\
\hline$\pi \pi^{*}\left(\mathrm{~L}_{\mathrm{b}}\right)$ & 0 & 2.2 & 0.04 & 0 & 5.4 & 0.05 \\
$\pi \pi^{*}\left(\mathrm{~L}_{\mathrm{a}}\right)$ & 3534 & 5.0 & 0.09 & 1828 & 6.3 & 0.12 \\
$\pi \sigma^{*}$ & 13727 & 7.3 & 0.00 & 12552 & 7.6 & 0.00
\end{tabular}

been employed. Three fundamentally different binding motifs can be distinguished: $\mathrm{NH} \cdots \mathrm{O}$ hydrogen bond structures (1a and $\mathbf{1 b}$ in Fig. 1 and Table 3), CN $\cdots$ H hydrogen bond structures ( 2 and 3 in Fig. 1 and Table 3), and $\pi$-bond van der Waals structures. No stable van der Waals bound minimum structure at the CC2-cc-pVTZ level of theory has been found. All structures have been optimized both using genuine CC2, as well as SCS-CC2. Similar resulting structures have been found with both methods, with the exception that at the SCS-CC2 level no stable structure $\mathbf{1 b}$ in the $S_{1}$ state has been found.

The stabilization energies, including zero-point energy (ZPE) corrections and considering the BSSE, are given in Table 4. Both $\mathrm{NH} \cdots \mathrm{O}$ hydrogen bond structures are by about 7 and $6 \mathrm{~kJ} \mathrm{~mol}^{-1}$ more stable than the $\mathrm{CN} \cdots \mathrm{H}$ hydrogen bond structures. The stabilization energies of $\mathbf{1 a}$ and $\mathbf{1 b}$ are too close to make a definite statement as to which of the two cluster structures is the more stable one. Both cluster structures show a slightly larger stabilization in the excited state and slight red shifts of their adiabatic excitation energies relative to that of the monomer. The origin of conformer $1 \mathrm{a}$ is red-shifted by $16 \mathrm{~cm}^{-1}$, and $\mathbf{1 b}$ shows a redshift of $21 \mathrm{~cm}^{-1}$. The experimental value is $51 \mathrm{~cm}^{-1}, c f$. Section 4.1 .

The orientation of the transition dipole moment (Fig. 1), as well as the main contributions to the excitation to the lowest excited singlet state of $0.81($ LUMO $\leftarrow$ HOMO-1) + 0.41(LUMO $+1 \leftarrow$ HOMO) $-0.27($ LUMO $+2 \leftarrow$ HOMO), $c f$. Fig. S1 of the ESI $\dagger$, are indicative for a transition to the ${ }^{1} \mathrm{~L}_{\mathrm{b}}$ state also for the 1:1 water cluster. 


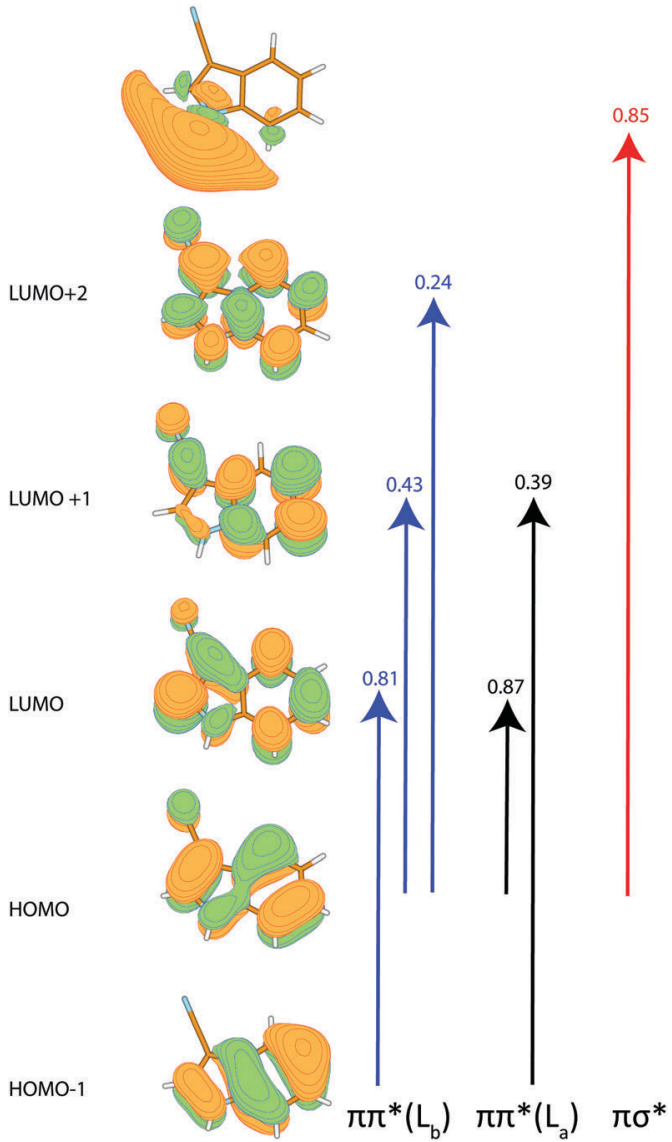

Fig. 3 Molecular orbitals of 3-cyanoindole with the coefficients of the $\pi \pi^{*}$ $\left(L_{b}\right)$ (blue), $\pi \pi^{*}\left(L_{a}\right)$ (black) and $\pi \sigma^{*}$ (red) excitations according to SCS-CC2/ cc-pVTZ calculations.

Table 3 SCS-CC2/cc-pVTZ computed and experimental molecular parameters of different 1:13-cyanoindole-water complexes. For details see text

\begin{tabular}{|c|c|c|c|c|c|c|c|}
\hline & \multicolumn{4}{|c|}{$\underline{\text { SCS CC2/cc-pVTZ }}$} & \multicolumn{3}{|c|}{ Experiment } \\
\hline & $1 \mathrm{a}$ & $1 b$ & 2 & 3 & $\sigma=1$ & $\sigma=0$ & $\Delta \sigma$ \\
\hline$A^{\prime \prime} / \mathrm{MHz}$ & 1083 & 1093 & 1292 & 2015 & 1104.10 & 1100.04 & -4.06 \\
\hline$B^{\prime \prime} / \mathrm{MHz}$ & 745 & 743 & 739 & 559 & 728.29 & 728.01 & -0.28 \\
\hline$C^{\prime \prime} / \mathrm{MHz}$ & 442 & 443 & 471 & 439 & 439.07 & 438.99 & -0.08 \\
\hline$\Delta I^{\prime \prime} / \mathrm{amu} \AA^{2}$ & -2.33 & -2.33 & -1.92 & -2.06 & -0.6380 & -2.3774 & \\
\hline$A^{\prime} / \mathrm{MHz}$ & 1091 & 1092 & 1279 & 1979 & 1099.63 & 1096.97 & -2.66 \\
\hline$B^{\prime} / \mathrm{MHz}$ & 734 & 734 & 731 & 554 & 724.63 & 724.33 & -0.30 \\
\hline$C^{\prime} / \mathrm{MHz}$ & 440 & 440 & 466 & 434 & 437.28 & 437.20 & 0.00 \\
\hline$\Delta I^{\prime} / \mathrm{amu} \AA^{2}$ & -2.56 & -3.25 & -1.90 & -2.46 & -1.2938 & -2.0979 & \\
\hline$\Delta A / \mathrm{MHz}$ & 8.10 & -2.33 & -12.85 & -36.19 & -4.47 & -3.07 & \\
\hline$\Delta B / \mathrm{MHz}$ & -10.95 & -8.79 & -7.70 & -5.44 & -3.65 & -3.68 & \\
\hline$\Delta C / \mathrm{MHz}$ & -2.56 & -3.25 & -4.84 & -4.92 & -1.79 & -1.79 & \\
\hline$\Delta \nu_{\text {Lor. }} / \mathrm{MHz}$ & - & - & - & - & 43.51 & 43.51 & \\
\hline$\tau / \mathrm{ns}$ & - & - & - & - & 3.66 & 3.66 & \\
\hline$\theta /^{\circ}$ & -39 & -39 & -26 & +13 & \pm 48.26 & \pm 48.26 & \\
\hline$\nu_{0} / \mathrm{cm}^{-1}$ & 36029 & 36024 & 35980 & 36140 & 35262.2 & 35261.9 & \\
\hline$\Delta \nu_{0} / \mathrm{MHz}$ & - & - & - & - & -8977.46 & & \\
\hline
\end{tabular}

\subsection{Experimental results}

3.2.1 3-Cyanoindole. Fig. 4 shows the rotationally resolved electronic spectrum of the origin of 3-cyanoindole at $35299.36 \mathrm{~cm}^{-1}$ ( 0 on the scale of the figure) along with the best
Table 4 Summary of stabilization energies (including ZPE and BSSE corrections) and adiabatic excitation (including ZPE) and vertical excitation and emission energies of the four most stable isomers of the 3-cyanoindolewater cluster at the SCS CC2/cc-pVTZ level of theory

\begin{tabular}{lllll}
\hline & $\mathbf{1 a}$ & $\mathbf{1 b}$ & $\mathbf{2}$ & $\mathbf{3}$ \\
\hline$\Delta E_{\text {stab. }}\left(\mathrm{S}_{0}\right) / \mathrm{kJ} \mathrm{mol}^{-1}$ & -28.46 & -28.35 & -20.41 & -21.86 \\
$\Delta E_{\text {stab. }}\left(\mathrm{S}_{0}\right) / \mathrm{cm}^{-1}$ & -2378.7 & -2370.2 & -1706.4 & -2122.01 \\
$\Delta E_{\text {stab. }}\left(\mathrm{S}_{1}\right) / \mathrm{kJ} \mathrm{mol}^{-1}$ & -28.64 & -28.61 & -21.18 & -20.71 \\
$\Delta E_{\text {stab. }}\left(\mathrm{S}_{1}\right) / \mathrm{cm}^{-1}$ & -2379.9 & -2391.3 & -1770.7 & -1731.2 \\
$\Delta E_{\text {adiabatic }} / \mathrm{cm}^{-1}$ & 36029 & 36024 & 35980 & 36140 \\
$\Delta E_{\text {vertical }}$ )opt. $\mathrm{S}_{0}$ & 38832 & 38870 & 38884 & 38940 \\
$\Delta E_{\text {vertical }} @ o p t . \mathrm{S}_{1}$ & 35843 & 35842 & 35735 & 36009
\end{tabular}

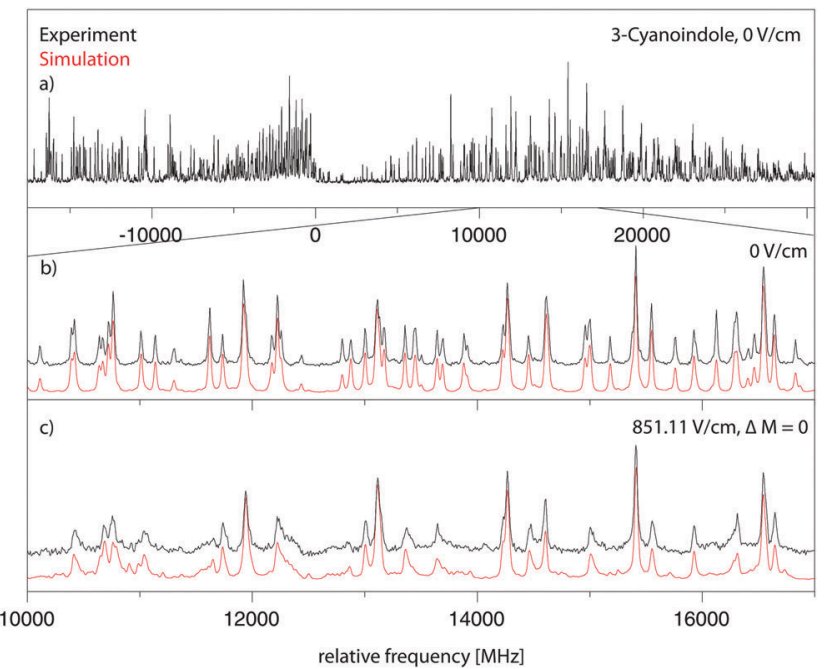

Fig. 4 Rotationally resolved electronic spectrum of the electronic origin of 3-cyanoindole at zero field and at $851.11 \mathrm{~V} \mathrm{~cm}^{-1}$ with $\Delta M=0$ selection rules, along with a simulation with the best CMA-ES fit parameters.

fit at zero field (trace (b)) and at a field strength of $851.11 \mathrm{~V} \mathrm{~cm}^{-1}$ (trace (c)). The electric field in the chosen set-up is parallel to the polarization of the plane of the exciting light, thus $\Delta M=0$ selection rules for the Stark spectrum hold. The spectrum was fit using a CMA-ES; the molecular parameters from the fit are summarized in Table 1 and are compared to the results of the SCS-CC2/cc-pVTZ calculations.

The origin band is an ab-hybrid with predominant a-type character. The exact analysis yields an angle of $\pm 15.3^{\circ}$. The indeterminacy of the TDM orientation can be resolved by comparison to the $a b$ initio computed value of $+4^{\circ}$. Thus, the orientation as shown in Fig. 1 seems to be the experimentally observed one. A more reliable determination can be performed on the basis of the TDM angle in the deuterated isotopologue of 3 -cyanoindole, and on the basis of the relative orientation of the transition moment and axis reorientation angle, which will be presented in Section 4.1.

A Lorentzian linewidth of $16.31 \mathrm{MHz}$ corresponds to an excited state lifetime of $9.8 \mathrm{~ns}$.

3.2.2 $\mathrm{N}$-Deuterated 3-cyanoindole. The knowledge of the position of the pyrrolic amino hydrogen in the inertial frame of 3-cyanoindole is crucial for the determination of the hydrogen 


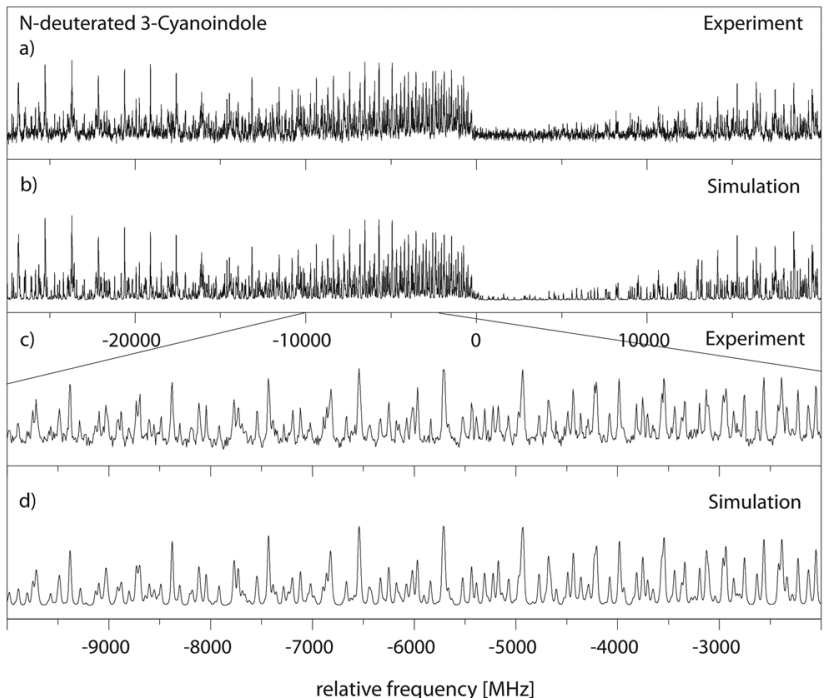

Fig. 5 Rotationally resolved electronic spectrum of the electronic origin of $\mathrm{N}$-deuterated 3 -cyanoindole, along with a simulation with the best CMA-ES fit parameters.

bond length in the 3-cyanoindole-water cluster. Therefore, we measured the spectrum of the N-D isotopologue, which allows for the determination of the hydrogen coordinates through application of Kraitchman's equations. Fig. 5 shows the rotationally resolved electronic spectrum of the origin of 3-cyanoindole- $\left(\mathrm{d}_{1}\right)$ at $35306.58 \mathrm{~cm}^{-1}$ ( 0 on the scale of the figure). The spectrum is composed mainly of a-type bands similar to the undeuterated species. The results of the CMA-ES fit of the spectrum are summarized in Table 1, where they are compared to the results of the SCS-CC2/cc-pVTZ calculations. The excited state lifetime of $14.8 \mathrm{~ns}$ is considerably longer than that of undeuterated 3-cyanoindole.

3.2.3 3-Cyanoindole-water. Fig. 6 presents the rotationally resolved electronic spectrum of the origin of the binary 3-cyanoindole-water cluster at $35262.2 \mathrm{~cm}^{-1}$. This origin band is redshifted by $51 \mathrm{~cm}^{-1}$ relative to the origin of 3-cyanoindole at $35299.36 \mathrm{~cm}^{-1}$. The spectrum is split into two subbands due to the large amplitude internal motion of the water moiety. The molecular symmetry group which takes this motion into account is $\mathrm{G}_{4}$, isomorphic with the point group $C_{2 \mathrm{v}}$. The lowest torsional state (with $\sigma=0$ ) is of $\mathrm{A}_{1}$ symmetry, and the energetically following (with $\sigma=1$ ) of $\mathrm{B}_{2}$ symmetry. Both subbands are fit using rigid asymmetric rotor selection rules, with the difference of the vibronic origins as an additional parameter. Trace (a) of Fig. 6 shows the experimental spectrum, and trace (b) the simulation using the best fit parameters from Table 3. The following traces (c) and (d) give the individual simulations for the two torsional subbands, due to the internal rotation of the water moiety. The resulting molecular parameters are given in Table 3. The spectrum is an ab-hybrid band with $56 \%$ b-type and $44 \%$ a-type character, and is split into two subbands by the internal rotation of the water moiety. The splitting between the origins of the two sub-bands amounts to $8977 \mathrm{MHz}$. The Lorentzian linewidth was determined to be $43 \mathrm{MHz}$, leading to an excited state lifetime of $3.6 \mathrm{~ns}$.

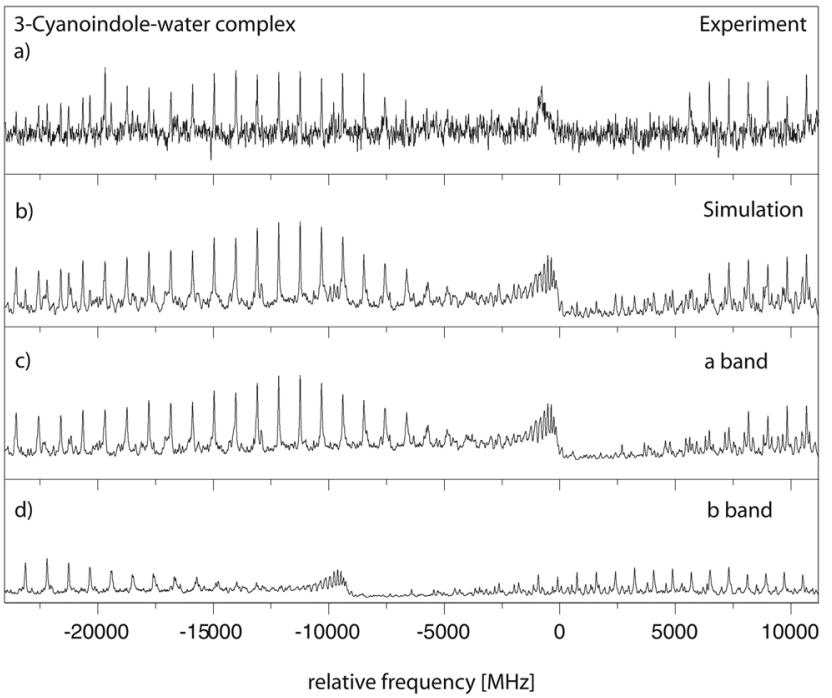

Fig. 6 Rotationally resolved spectrum of the electronic origin of the 3-cyanoindole-water cluster, along with a simulation using the best CMA-ES fit parameters.

3.2.4 Excited state lifetimes. Since the question of excited state lifetimes is crucial for the discussion of the photophysical properties of 3-cyanoindole, we performed lifetime measurements of 3-cyanoindole and of 5-cyanoindole and indole in different solvents using time correlated single photon counting. Fig. 7 presents the decay curves for indole, 3-cyanoindole, and 5-cyanoindole in ethyl acetate. The excited state lifetimes of isolated indole, 5-cyanoindole, 3-cyanoindole, and 3-cyanoindole $\left(\mathrm{d}_{1}\right)$ in ethyl acetate (EA) and of 3-cyanoindole $\left(\mathrm{d}_{1}\right)$ in $\mathrm{D}_{2} \mathrm{O}$ are summarized in Table 5.

\section{Discussion}

\subsection{3-Cyanoindole}

4.1.1 Geometric and electronic structure of the excited state. The small negative inertial defects of 3-cyanoindole in the electronic ground state $\left(-0.02 \mathrm{amu} \AA^{2}\right)$ and in the lowest electronically excited state $\left(-0.08\right.$ amu $\left.\AA^{2}\right)$ show its planarity in both electronic states. In contrast to indole and 5-cyanoindole, the inertial defect decreases slightly upon excitation to the $S_{1}$ state.

The angle of the TDM with the inertial $a$-axis was determined experimentally to be $\pm 15.3^{\circ}$. In the following we will show two independent determinations of the sign of the TDM angle, which do rely on the comparison to the calculated TDM orientation.

The geometry change upon electronic excitation causes a reorientation of the inertial axes of a molecule if its symmetry is sufficiently low. ${ }^{56}$ The molecule fixed coordinate system, which rotates with the molecule, and the space fixed coordinate system can be interconverted by the Euler rotation matrix. In the case of different geometries of the two states, which are connected by the spectroscopic transition, two moving axis systems, and consequently two sets of Eulerian angles $\theta, \phi$, and $\chi$ are needed, one for each state. In this way, the rotational 


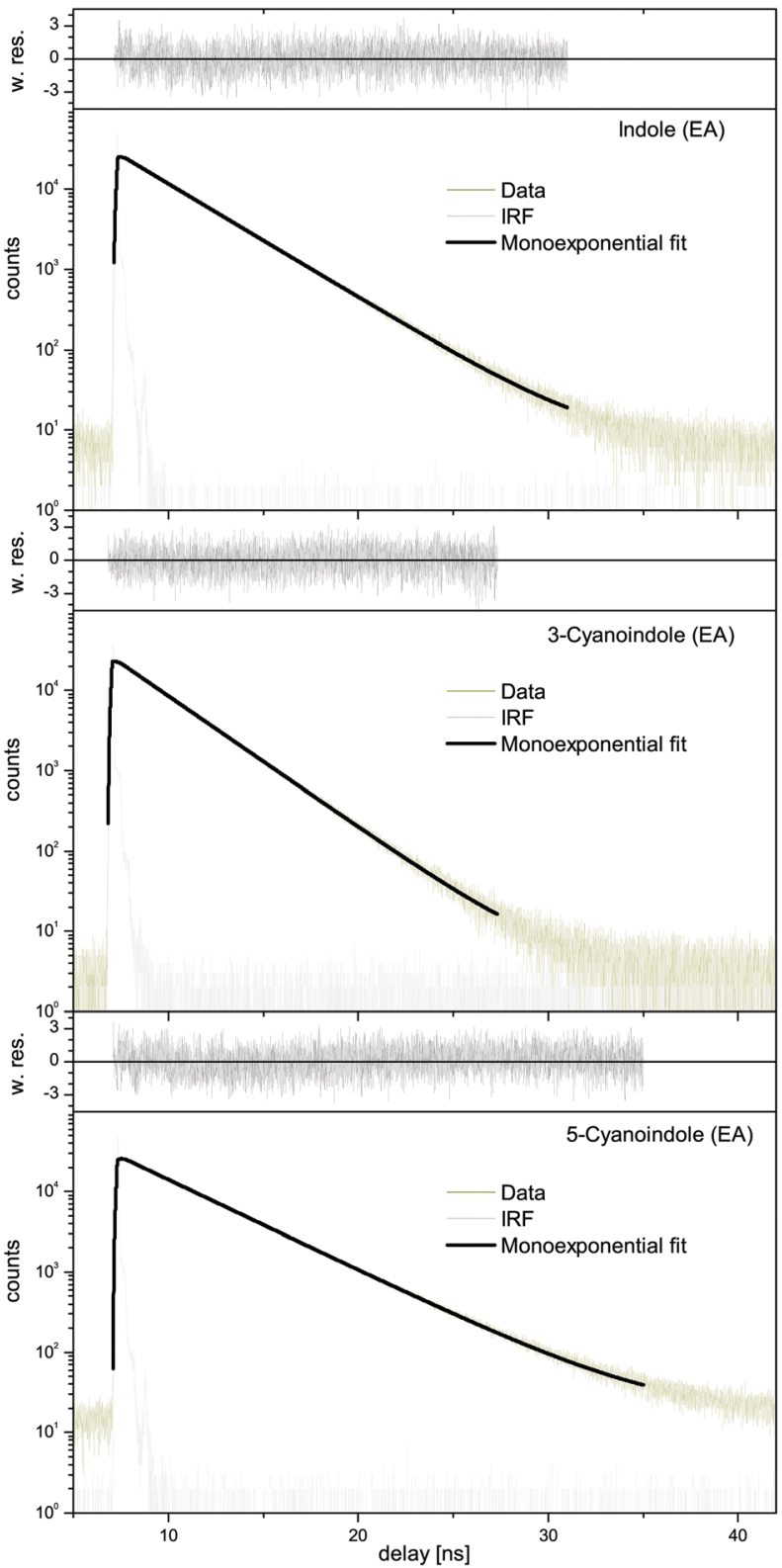

Fig. 7 TCSPC of indole, 3-cyanoindole, and 5-cyanoindole in ethyl acetate (EA) with monoexponential fits and weighted residuals (w. res.). The instrument response function (IRF) at the respective excitation wavelength is shown in light gray.

selection rules, which are valid only for unchanged geometries, have to be modified. Hougen and Watson's approach to this problem is the diagonalization of the rotational Hamiltonian for each state separately, and a rotation of the eigenvectors through a rotation matrix containing the axis reorientation angles $\theta_{\mathrm{T}}, \phi_{\mathrm{T}}$, and $\chi_{\mathrm{T}}$. Pratt and coworkers introduced a different procedure. ${ }^{57}$ In spite of rotating the wave functions, they express the Hamiltonian of the excited state in the coordinates of the ground state. Thus, the rotation of the eigenvectors is replaced by a rotation of the Hamiltonian about the axis reorientation angles $\theta_{\mathrm{T}}, \phi_{\mathrm{T}}$, and $\chi_{\mathrm{T}}$. This approach has the big advantage that the similarity transformation has to be applied only once, while
Table 5 Angles $\theta$ of the orientation of the transition dipole moment and excited state lifetimes (molecular beam and solution) of 3-cyanoindole (3-Cl), 5-cyanoindole (5- $\mathrm{Cl}$ ) and the 3-cyanoindole-water cluster. In contrast to the $\theta$ values shown in Table 1, those given here are given with respect to the pseudo $C_{2}$-axis of indole

\begin{tabular}{llllll}
\hline & Indole & $3-\mathrm{CI}$ & $3-\mathrm{CI}\left(\mathrm{d}_{1}\right)$ & 5-CI & 3-CI- $\mathrm{H}_{2} \mathrm{O}$ \\
\hline$\theta /^{\circ}$ & $+38^{12}$ & +35 & +36 & $-12^{22}$ & +51 \\
$\tau / \mathrm{ns}$ & $17.6^{12}$ & 9.8 & 14.8 & $12^{22}$ & 3.9 \\
$\tau(\mathrm{EA})^{\text {solv. }} / \mathrm{ns}$ & $3.1^{c}$ & $2.6^{a}$ & $2.7^{a}$ & $3.6^{d}$ & - \\
$\tau\left(\mathrm{H}_{2} \mathrm{O}\right)^{\text {solv. }} / \mathrm{ns}$ & $4.5^{19}$ & $<0.05^{19}$ & $<0.02^{b}$ & $0.3^{19}$ & -
\end{tabular}

${ }^{a}$ Excitation of 3-cyanoindole and 3-cyanoindole $\left(\mathrm{d}_{1}\right)$ in ethyl acetate (EA) at $283 \mathrm{~nm} .{ }^{b} 3-\mathrm{CI}\left(\mathrm{d}_{1}\right)$ has been measured in $\mathrm{D}_{2} \mathrm{O}$ with excitation at $283 \mathrm{~nm}$. ${ }^{c}$ Excitation of indole in EA at $284 \mathrm{~nm} .{ }^{d}$ Excitation of 5-cyanoindole in $\mathrm{EA}$ at $295 \mathrm{~nm}$.

in the Hougen-Watson approach, each $J$ block has to be rotated by means of a $(2 J+1) \times(2 J+1)$ rotation matrix. When rotated, the excited state Hamiltonian has no longer the four group symmetry of the ground state. Thus, the effect of axis reorientation is a change of the intensities of several rovibronic lines. The line positions however are not altered, since the rotated and unrotated eigenfunctions are related though a similarity transformation. Thus, the eigenvalues of the rotated and unrotated Hamiltonian are the same. ${ }^{57}$

Pratt and coworkers have shown how the relative orientation of the transition dipole moment $\theta$ and the axis reorientation $\theta_{\mathrm{T}}$ can be used to remove the indeterminacy of the sign of $\theta$. The combinations of $+\theta /+\theta_{\mathrm{T}}$ and $-\theta /-\theta_{\mathrm{T}}$ have the same intensity pattern, which in turn is different for the $+\theta /-\theta_{\mathrm{T}}$ and $-\theta /+\theta_{\mathrm{T}}$ combinations. From a fit of the rovibronic intensities to the signed value of the axis reorientation angle, it can be deduced if TDM and axis reorientation have the same or opposite signs.

From the SCS-CC2 optimized structures, the axis reorientation angle of the inertial axis system upon electronic excitation $\theta_{\mathrm{T}}$ can be determined using the relation for planar molecules given by Hougen and Watson: ${ }^{56}$

$$
\tan \left(\theta_{\mathrm{T}}\right)=\frac{\sum_{i} m_{i}\left(a_{i}^{\prime} b_{i}^{\prime \prime}-b_{i}^{\prime} a_{i}^{\prime \prime}\right)}{\sum_{i} m_{i}\left(a_{i}^{\prime} a_{i}^{\prime \prime}+b_{i}^{\prime} b_{i}^{\prime \prime}\right)}
$$

Here, the doubly primed coordinates refer to the principal axis system in the electronic ground state and the singly primed quantities to the respective excited state inertial system and the $m_{i}$ are the atomic masses. Using the SCS-CC2 optimized structures for the ${ }^{1} \mathrm{~L}_{\mathrm{b}}$ state, we obtain an axis reorientation angle $\theta_{\mathrm{T}}$ of $-0.45^{\circ}$ for the ${ }^{1} \mathrm{~L}_{\mathrm{b}}$ state. Since the optimization of the ${ }^{1} \mathrm{~L}_{\mathrm{a}}$ state ran into a conical intersection at the SCS-CC2 level of theory (vide supra), the TD-DFT optimized geometry was used and an angle of $0.99^{\circ}$ was obtained for the ${ }^{1} \mathrm{~L}_{\mathrm{a}}$ state. The calculated value for the ${ }^{1} \mathrm{~L}_{\mathrm{b}}$ state is in good agreement with the value from the fit of the spectrum using the axis reorientation Hamiltonian of $0.52^{\circ}$.

Since axis reorientation has no effect on the eigenvalues, i.e. the energies of the rovibrational levels, the only quantity to be fit is the axis reorientation angle $\theta_{\mathrm{T}}$, leaving all other parameters at their before determined values. However, only a few lines are 
considerably affected by axis reorientation. Improvement of the cost function in the course of the optimization might therefore be concealed by the majority of lines whose intensities do change. In order to circumvent this problem, we included only sections of the spectrum in the fit, which show, guided by a preliminary simulation, strong intensity variations. Two kinds of solutions were obtained. The combination of $\theta$ positive and $\theta_{\mathrm{T}}$ negative as well as $\theta$ negative and $\theta_{\mathrm{T}}$ positive had a better cost function than the combination of both angles positive or both angles negative. Therefore, we know $\theta_{\mathrm{T}}$ and $\theta$ must have different signs. Using the fact that $\theta_{\mathrm{T}}$ is negative from the above considerations, $\theta$ must be positive.

To facilitate the comparison to other indoles, we transform the angle of the TDM to refer to the pseudo $C_{2}$-axis of indole. The results are shown in Table 5. Clearly, the orientation of the TDM is the same as in indole, and the lowest excited singlet state of 3-cyanoindole can be assigned to an ${ }^{1} \mathrm{~L}_{\mathrm{b}}$ like state, in contrast to 5-cyanoindole in which the opposite sign of the TDM points to an ${ }^{1} \mathrm{~L}_{\mathrm{a}}$ state.

The fact that no stable minimum at the SCS-CC2 level could be located for the ${ }^{1} \mathrm{~L}_{\mathrm{a}}$ state might arise from two different reasons. (i) The ${ }^{1} L_{a}$ minimum might be so far outside the Franck-Condon region, that our searches just missed the minimum structure and followed instead the falling ${ }^{1} \mathrm{~L}_{\mathrm{a}}$ potential right into the conical intersection (CI) with ${ }^{1} \mathrm{~L}_{\mathrm{b}}$. Although we tried a plethora of starting geometries, which were constructed from the known ${ }^{1} \mathrm{~L}_{\mathrm{a}}$ geometries of indole and 5-cyanoindole, we cannot completely exclude this reason. (ii) A much more probable explanation for this finding is a very shallow ${ }^{1} \mathrm{~L}_{\mathrm{a}}$ minimum in the coordinate, which connects ${ }^{1} \mathrm{~L}_{\mathrm{a}}$ and ${ }^{1} \mathrm{~L}_{\mathrm{b}}$ through the CI. Such a case was found for tryptamine. ${ }^{58}$ The slightest deviations from the correct ${ }^{1} \mathrm{~L}_{\mathrm{a}}$ geometry at the chosen level of theory will then cause the optimization to run into the CI.

4.1.2 Excited state lifetimes. The $S_{1}$ state lifetime of the isolated 3-cyanoindole was determined to be $9.8 \mathrm{~ns}$, which is shorter than the respective lifetimes of indole $(17 \mathrm{~ns})^{12}$ and 5 -cyanoindole (12 ns). ${ }^{22}$ In different solvents a similar trend for the lifetimes of indole and of the cyanoindoles was found. Independent of the solvent, the fluorescence lifetime of 3-cyanoindole was always shorter than that of 5-cyanoindole and of indole. ${ }^{19}$ For the isolated $1: 1$ water complex the shortest lifetime of $3.9 \mathrm{~ns}$ has been measured, $c f$. Table 5 . Interestingly, also the lifetime of 3-cyanoindole in water solution is the shortest $(<0.05 \mathrm{~ns}){ }^{19}$ followed by the aqueous solution of 5 -cyanoindole $(0.3 \mathrm{~ns})^{19}$ and indole $(4.5 \mathrm{~ns}){ }^{19}$ The lifetime of deuterated 3-cyanoindole in the gas phase is longer than that of the undeuterated isotopologue (14.8 vs. $9.8 \mathrm{~ns}$ ). A similar trend has been found for phenol (13.3 ns vs. $2.4 \mathrm{~ns}$ ). Sobolewski and Domcke have shown, that along with the lowest two excited singlet ${ }^{1} \mathrm{~L}_{\mathrm{a}}$ and ${ }^{1} \mathrm{~L}_{\mathrm{b}}$ states, which are of $\pi \pi^{*}$ character, a third state of $\pi \sigma^{*}$ character plays a crucial role in the photophysics of these chromophores. ${ }^{17,53-55}$ This $\pi \sigma^{*}$ state is repulsive along the $\mathrm{OH}$ coordinate for phenol and along the $\mathrm{NH}$ coordinate for indole and forms conical intersections with the directly excited state and subsequently with the electronic ground state. For excitations above the threshold, a rapid decay channel is open,

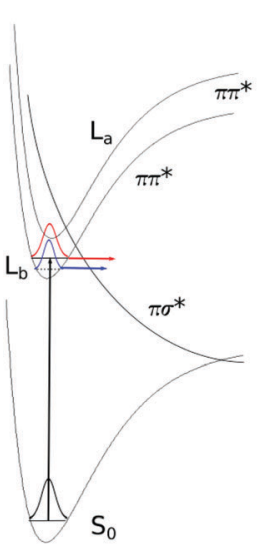

a

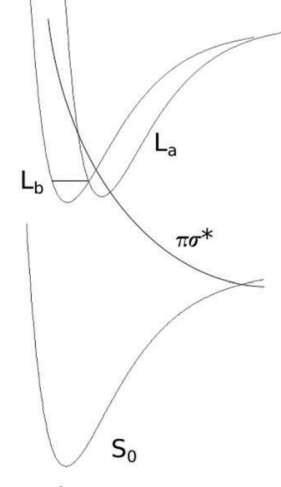

b
C

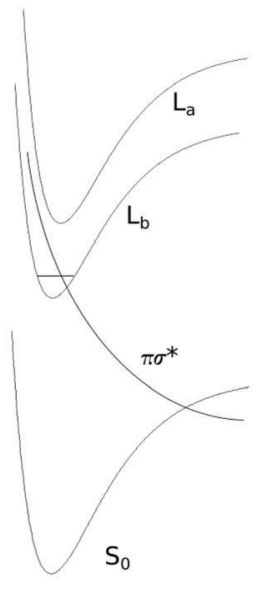

Fig. 8 Schematic potential energy profiles along the $\mathrm{NH}$ stretch coordinate. (a) The conical intersection between $\pi \pi^{\star}$ and $\pi \sigma^{*}$ above the zeropoint energy level of the excited state. The dashed line represents the zero-point energy level of the $\mathrm{N}$-deuterated isotopologue. The wave packet prepared at the zero-point level (in red for the NH isotopologue, in blue for the ND isotopologue) tunnels through the barrier, formed by the $\pi \pi^{\star}-\pi \sigma^{\star}$ conical intersection. (b) The same situation as in (a) but the minimum of the second $\pi \pi^{\star}$ is shifted and forms a new $\mathrm{Cl}$ with the lower $\pi \pi^{\star}$ state. (c) The $\pi \sigma^{\star}$ state is further stabilized with respect to the $\pi \pi^{\star}$ state. The zero point energy level is above the conical intersection.

which connects the primarily excited $\pi \pi^{*}$ state through a conical intersection (CI) with the $\pi \sigma^{*}$ state, and subsequently through a second CI with the ground state. The $\pi \pi^{*}-\pi \sigma^{*}$ CI induces a barrier on the $S_{1}$ potential energy surfaces, through which a wave packet prepared at the zero-point level of the primarily excited state can tunnel. This causes the $S_{1}$ state lifetime of phenol to be as short as $2.4 \mathrm{~ns}$, while the lifetime of deuterated phenol is considerably longer (13.3 ns) due to the lower zero-point energy. The same behavior is found for 3-cyanoindole and its ND-deuterated isotopologue (Fig. 8a). The isotope effect on the excited state lifetime of indole is negligible (17.6 ns vs. $17.2 \mathrm{~ns}$ for the deuterated isotopologue ${ }^{59}$ ), a consequence of the larger barrier formed by the $\pi \pi^{*}-\pi \sigma^{*}$ CI in indole. We therefore claim a lower barrier, formed on the $S_{1}$ potential energy surface, to be responsible for the shorter lifetime of 3-cyanoindol compared to indole and 5-cyanoindole. The calculation of the vertically excited $\pi \pi^{*}$ and $\pi \sigma^{*}$ states in Section 3.1 strongly supports this suggestion.

For the water clusters in the gas phase, only lifetimes for indole- $\mathrm{H}_{2} \mathrm{O}$ and 3-cyanoindole- $\mathrm{H}_{2} \mathrm{O}$ (3.6 ns) are known experimentally. Indole- $\mathrm{H}_{2} \mathrm{O}$ however, is hard to compare, because two very different values are reported. Korter et al. determined a value of $5 \mathrm{~ns}$ from the Lorentz component to the full linewidth in a rotationally resolved electronic spectrum of the origin band. ${ }^{25}$ Arnold and Sulkes found a value of 21 ns using timecorrelated single photon counting after excitation of the electronic origin. ${ }^{60}$ What is then the reason for the short lifetime in the $1: 1$ cluster and the ultrashort lifetime in the aqueous solution of 3-cyanoindole? The answer to this problem is further complicated by the fact that depending on the relative stabilization of the different excited states, conical intersections 
not only between the $\pi \pi^{*}$ and the $\pi \sigma^{*}$ states develop, but also between the lowest $\pi \pi^{*}{ }^{1} \mathrm{~L}_{\mathrm{a}}$ and ${ }^{1} \mathrm{~L}_{\mathrm{b}}$ states ${ }^{58}$ ( $c f$. Fig. 8b). Regarding the fact that the $\pi \pi^{*}-\pi \sigma^{*}$ barrier of 3-cyanoindole is lower than that of indole (vide supra), it seems plausible that the $\pi \sigma^{*}$ potential is lowered even further through stabilization via the water dipole (Fig. 8c). The short lifetime would then originate from the increase of the nonradiative decay rate. A conclusive answer, however, can only be given if the oscillator strengths to the fully adiabatically optimized excited states along the dissociative NH coordinate are known. These calculations are currently under way.

4.1.3 Dipole moments. The ground state dipole moment of $5.90 \mathrm{D}$ decreases to $5.35 \mathrm{D}$ in the excited singlet state. The angle of the dipole moment with the inertial $a$-axis slightly decreases from 45 to $39^{\circ}$ upon excitation. For bare indole, values of $1.963 \mathrm{D}\left(\mathrm{S}_{0}\right)$ and $1.856 \mathrm{D}\left(\mathrm{S}_{1}\right)$ with angles to the $a$-axis of 45 and $33^{\circ}$, respectively, have been reported. ${ }^{9}$ However, one has to keep in mind that the heavy cyano group rotates the inertial $a$ axis by $36^{\circ}$ in the molecular frame as shown in Fig. 9.

For 5-cyanoindole we found a good additivity of the dipole moments of indole and of the cyano fragment in the electronic ground state. ${ }^{61}$ In this molecule, the indole dipole and the dipole of the cyano fragment which is attached to the benzenoid ring are nearly aligned. For 3-cyanoindole, the individual dipoles form a large angle, and the cyano fragment is attached to the pyrrolic ring. Fig. 9b shows the experimental dipole moment vector of 3-cyanoindole (red, straight vector) and of indole (black, straight vector). Subtracting the experimentally determined dipole moment vector of 3-cyanoindole from that of indole ${ }^{9}$ should lead to the dipole moment vector of the substituent $\left(\vec{\mu}_{\mathrm{CN}}=\vec{\mu}_{3 \mathrm{CN} \text {-indole }}-\vec{\mu}_{\text {indole }}\right)$. If the dipole moments of indole and the cyano fragment are additive like in 5-cyanoindole, the difference vector will point in the direction of the fragment dipole with the corresponding length of the fragment dipole, $c f$. Fig. 9b and c. The most appropriate dipole moments to mimic the fragment dipole of the cyano group, attached to the pyrrole ring, are those of methyl cyanide $\mathrm{CH}_{3} \mathrm{CN}$, isopropyl cyanide $\left(\mathrm{CH}_{3}\right) \mathrm{CHCN}$ and benzonitrile $\mathrm{C}_{6} \mathrm{H}_{5} \mathrm{CN}$. The ground state dipole moment of methyl cyanide was determined by Steiner and Gordy to be $3.913 \mathrm{D},{ }^{62}$ and the dipole moment of isopropyl cyanide has been determined to be 4.07 D by Müller et al. ${ }^{63}$ The most exact value of the benzonitrile dipole moment was reported by Wohlfahrt et al. to be $4.5152 \mathrm{D}^{31}$ Thus, a value of around $4 \mathrm{D}$ for the cyano fragment, colinear with the $\mathrm{CN}$ bond, can be expected. Inspection of Fig. 9b shows that the fragment dipole indeed points in the same direction as the cyano fragment dipole. From the vectorial difference of the ground state dipoles a fragment dipole moment of $5.5 \mathrm{D}$ is predicted, which is more than $1 \mathrm{D}$ larger than expected from a simple additivity model. Using the excited state dipole moments of indole and 3-cyanoindole, a value of 5.6 D results, in agreement with the fragment dipole determined from the ground state values. Inspection of the direction of the fragment dipole in Fig. 9c however shows that in the excited state, even the direction of the dipole moment deviates from that of the fragment.

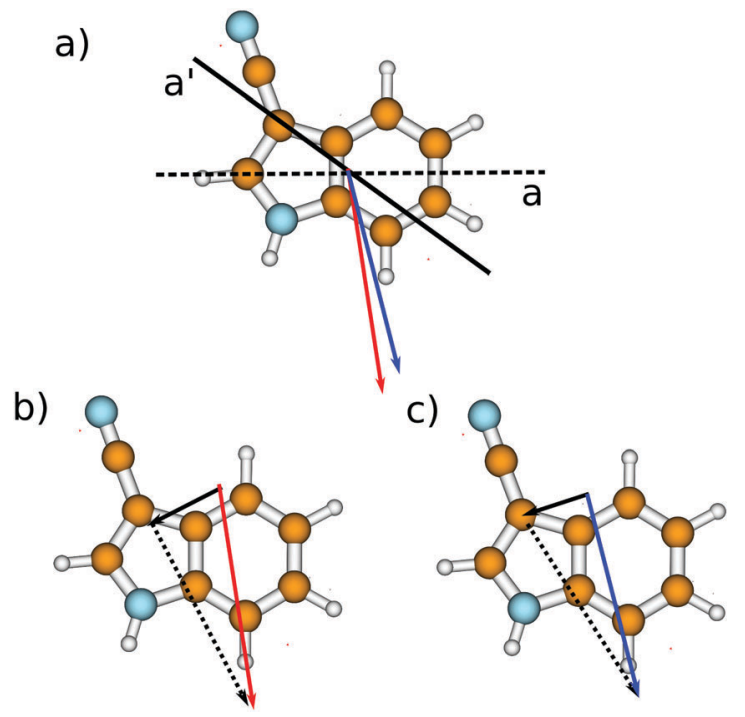

Fig. 9 (a) Inertial a-axis of indole (dashed line) and 3-cyanoindole (straight line). The ground state dipole moment of 3 -cyanoindole is shown as a red vector with direction from - to +, and that of the excited state as a blue vector. (b) Ground state dipole moments of 3-cyanoindole (red, straight), and indole (black, straight) and the difference vector (black, dotted). (c) Excited state dipole moments of 3-cyanoindole (blue, straight), and indole (black, straight) and the difference vector (black, dotted). In all cases the dipole vectors are shifted from their position in the original molecule for the sake of clarity.

\subsection{3-Cyanoindole-water}

4.2.1 Barrier to water torsion. The origin spectrum of the 3cyanoindole-water cluster is split into two subbands, due to an internal motion of the water moiety in the cluster. The basic theory for this motion of the water moiety has been worked out in detail for phenol-water ${ }^{64}$ and indole-water. ${ }^{25}$ Therefore, we keep the theoretical description short; all necessary equations and their derivation can be found in ref. 65 . The motion of the water can be described as motion in a periodic two-fold potential. The rotational levels split into two subtorsional components, $\sigma=0$ and $\sigma=1$. This $\sigma$ is chosen such that it represents the symmetry of the torsional wavefunctions and therefore the torsional problem is diagonal in $\sigma$. The $\sigma=0$ levels have A symmetry and a spin statistical weight of 1 , while the $\sigma=1$ levels are of B symmetry with a spin statistical weight of 3 in the G4 molecular symmetry group. ${ }^{64} \Delta \sigma=0$ selection rules hold for the transitions from the electronic ground to the excited state. Since for the vibrational ground state (and for all even $v$ states) the $\sigma=0$ state is below the $\sigma=1$ state, the fact that the strong subtorsional band $(\sigma=1 \leftrightarrow \sigma=1)$ is at a shorter wavelength than the weak band ( $\sigma=0 \leftrightarrow \sigma=0)$ immediately shows that the barrier in the electronically excited state must be smaller than in the ground state. The energy difference of $8977.46 \mathrm{MHz}$ between these subtorsional bands is given by difference in the subtorsional splittings in the two electronic states $E_{\sigma=0}-E_{\sigma=1}$.

Further information is contained in the difference of the rotational constants between the $\sigma=0$ and $\sigma=1$ bands. Table 3 shows that in the electronic ground state mainly $A$ changes, 
while $B$ and $C$ are nearly the same for the subtorsional levels. Thus, the torsional axis is mostly parallel to the $a$ inertial axis in the cluster. The difference of the rotational constants between $\sigma=0$ and $\sigma=1$ bands $\left(\Delta B_{\mathrm{g}}=B_{\mathrm{g}}^{\sigma=0}-B_{\mathrm{g}}^{\sigma=1}\right)$ contains the second order perturbation terms, which can be used for an independent determination of the torsional barrier. A LevenbergMarquart fit of the subtorsional splitting and the differences of the rotational constants yield barriers of $148 \mathrm{~cm}^{-1}$ for the $S_{0}$ and of $125 \mathrm{~cm}^{-1}$ for the $S_{1}$ state, respectively, for a torsional constant of $15.2 \mathrm{~cm}^{-1}$.

4.2.2 Structure of the water cluster. The inertial defects $\Delta I$ of both torsional sub-bands of the 3-cyanoindole-water cluster in the ground and electronically excited state are small and negative $\left(\Delta I^{\prime \prime}=-0.6380\right.$ amu $\AA^{2}$ for the $\sigma=1$ subband and -2.3774 amu $\AA^{2}$ for $\sigma=0$ ) They are in the range of what is expected for a structure in which all heavy atoms are in-plane. For indole-water and phenol-water trans-linear hydrogen bond structures are found with inertial defects of -1.412 amu $\AA^{2}$ (indole-water) and $-2.086 \mathrm{amu} \AA^{2}$ (phenol-water). Thus, we conclude that also the 3-cyanoindole-water cluster forms a structure in which only the hydrogen atoms of the water moiety are located outside the plane of the chromophore in agreement with the structures of the computed most stable complexes (a and b in Fig. 1). Torsional excitation of the water averages the position of the water hydrogen atoms with respect to the plane. This explains why the inertial defect of the $\sigma=1$ component is smaller than that of the $\sigma=0$ component.

The position of the water molecule in the 3-cyanoindolewater cluster can be determined from the rotational constants of the water cluster and the monomer without further knowledge of the monomer structure using a Kraitchman ${ }^{66}$ analysis. The only assumption which is made therein is that the structure of the monomer moiety does not change upon cluster formation. While the original intention in using the Kraitchman equations is the determination of the Cartesian coordinates of an isotopically substituted atom in a molecule, it is also applicable in determining the center-of-mass (COM) distance of the two constituents of binary clusters. This procedure has first been applied to noble gas clusters of organic molecules, ${ }^{67}$ which directly yields the position of the noble gas atom in the inertial frame of the uncomplexed monomer. Later, it was extended to molecular clusters of hydrogen donors with water, in which the distance of the water COM from the COM of the organic molecule was determined. From the known structure of the water moiety, and the position of the oxygen (phenol) or nitrogen (indole) in the inertial frame of the monomer, also the heavy atom distance in hydrogen bonded clusters like $\mathrm{N}-\mathrm{H} \cdots \mathrm{O}$ or $\mathrm{O}-\mathrm{H} \cdots \mathrm{O}$ could be determined.

Using this method, we determined the distance between the COMs of the 3-cyanoindole monomer and of the water moiety in the ground state to be $494.1 \mathrm{pm}$ and in the electronically excited state to be $492.1 \mathrm{pm}$. The Kraitchman analysis for the amino hydrogen position in both the ground and excited states of 3-cyanoindole has been performed using the rotational constants of 3-cyanoindole and of the $\mathrm{N}$-deuterated 3-cyanoindole isotopologue from Table 1 . The $r_{0}$ coordinates of the
Table 6 Kraitchman $r_{0}$ coordinates of the pyrrolic amino hydrogen atom and of the water center of mass (COM) in pm

\begin{tabular}{|c|c|c|c|c|}
\hline \multirow[b]{2}{*}{ State } & \multicolumn{2}{|l|}{$\underline{\mathrm{S}_{0}}$} & \multicolumn{2}{|l|}{$\underline{S_{1}}$} \\
\hline & $\mathrm{NH}$ & Water & $\mathrm{NH}$ & Water \\
\hline$a$ & 7.4 & 155.3 & 7.6 & 157.2 \\
\hline$b$ & 285.3 & 469.1 & 283.9 & 466.3 \\
\hline$R$ & 285.4 & 494.1 & 284.0 & 492.1 \\
\hline NH $\cdots \mathrm{O}(\exp )$. & 201.9 & & 201.7 & \\
\hline $\mathrm{NH} \cdots \mathrm{O}$ (theo.) & 192.6 & & 192.3 & \\
\hline
\end{tabular}

$\mathrm{H}$-atom along with the $r_{0}$ coordinates of the water COM in the same reference frame are shown in Table 6.

From the so determined distance of the water COM from the amino hydrogen and the known distance of the COM of the water moiety from the water oxygen atom, the $\mathrm{NH} \cdots \mathrm{O}$ distance can be determined to be $201.9 \mathrm{pm}$ in the ground state and 201.7 in the excited state. This is the lower limit under the assumption that the amino hydrogen, water COM and oxygen atom are located on a straight line. If there is an angle $\alpha$ between the $\mathrm{HOH}$ plane and the hydrogen bond, the resulting bond length would be smaller by a factor of $\cos (\alpha)$. For both conformers 1a and $\mathbf{1 b}$ this angle is smaller than $10^{\circ}$, equivalent to a reduction of less than $3 \mathrm{pm}$. The obvious difference between the experimental and the $a b$ initio value for the hydrogen bond length results from zero-point vibrational averaging of the hydrogen bond along its very shallow stretching coordinate.

4.2.3 Electronic structure of the excited state. Table 5 shows that the orientation of the TDM vector of 3-cyanoindole-water, if transformed into the principal axis system of indole, is the same as in indole and in the 3-cyanoindole monomer, but opposite to that of 5-cyanoindole. The lowest excited state is therefore an ${ }^{1} \mathrm{~L}_{\mathrm{b}}$ like state as for the monomer. The reason for this deviant behavior can be found in the fact that for 3-cyanoindole the dipole moment in the ${ }^{1} \mathrm{~L}_{\mathrm{a}}$ state is smaller than in the ${ }^{1} \mathrm{~L}_{\mathrm{b}}$ state. All other indoles have a larger ${ }^{1} \mathrm{~L}_{\mathrm{a}}$ dipole moment and thus a stabilization of the ${ }^{1} \mathrm{~L}_{\mathrm{a}}$ state with respect to the ${ }^{1} \mathrm{~L}_{\mathrm{b}}$ state.

\section{Conclusions}

The dipole moment of 3-cyanoindole and its orientation in the ground and the lowest electronically excited state could be determined to be very similar. The excited state lifetime of 3 -cyanoindole is considerably shorter than that of indole and 5-cyanoindole with the 1:1 water cluster of 3-cyanoindole being especially short-lived. The electronic nature of the lowest excited state is a locally excited (LE) state with strong ${ }^{1} \mathrm{~L}_{\mathrm{b}}$ character. The adiabatically higher lying ${ }^{1} \mathrm{~L}_{\mathrm{a}}$ state has, in contrast to indole and 5-cyanoindole (and many other indole derivatives), a smaller permanent dipole moment. Therefore, the ${ }^{1} \mathrm{~L}_{\mathrm{b}}$ state is stabilized more strongly upon attachment of the polar water molecule than the ${ }^{1} \mathrm{~L}_{\mathrm{a}}$ state. This causes the $1: 1$ water cluster to be also of ${ }^{1} \mathrm{~L}_{\mathrm{b}}$ character. Although we can confirm the above statement only for the binary water cluster, one might speculate that the exceptionally short lifetime of 3-cyanoindole in water solution might be due to the fact that the emitting state is the ${ }^{1} \mathrm{~L}_{\mathrm{b}}$ which is 
different from the other cyanoindoles and indole in which ${ }^{1} \mathrm{~L}_{\mathrm{a}}$ emission is observed. ${ }^{14}$

The characterization of the emitting state could be performed using high resolution (Stark) spectroscopy for the first time. Permanent dipole moments, transition dipoles, fluorescence lifetimes and structures of the isolated molecule, as well as of the $1: 1$ water cluster (with the exception of the permanent dipole moments) could be obtained for the first time. These investigations show that already the first solvating water molecule opens the way to the unusually short life time of 3-cyanoindole in water solution. One might ask why we omitted the measurement of the Stark spectrum for the water cluster. Certainly, this would have added extremely important information about the function of the water moiety in the photophysics of the monomer. Unfortunately, 3-cyanoindole behaves differently than other cyanoindoles. Upon heating in the source chamber, 3-cyanoindole sublimates into an extremely fine powder, which clogs the orifice of the nozzle after a few minutes even at strongly elevated temperatures of the nozzle. Since the time for optimization of the cluster conditions was too short to get a better signal to noise ratio, we were unable to record the Stark spectrum.

\section{Conflicts of interest}

There are no conflicts to declare.

\section{Acknowledgements}

Financial support of the Deutsche Forschungsgemeinschaft via grant SCHM1043/12-3 is gratefully acknowledged. Computational support and infrastructure was provided by the "Center for Information and Media Technology" (ZIM) at the HeinrichHeine-University Düsseldorf (Germany). We furthermore thank the Regional Computing Center of the University of Cologne (RRZK) for providing computing time on the DFG-funded High Performance Computing (HPC) system CHEOPS as well as support. Financial support provided by CONACYT under the grant 277871 is greatly appreciated.

\section{References}

1 J. R. Platt, J. Chem. Phys., 1949, 17, 484-495.

2 G. Weber, Biochem. J., 1960, 75, 335-345.

3 T. B. C. Vu, I. Kalkman, W. L. Meerts, Y. N. Svartsov, C. Jacoby and M. Schmitt, J. Chem. Phys., 2008, 128, 214311.

4 B. Albinsson and B. Nordén, J. Phys. Chem., 1992, 96, 6204.

5 D. M. Sammeth, S. Yan, L. H. Spangler and P. R. Callis, J. Phys. Chem., 1990, 94, 7340.

6 T. L. O. Barstis, L. I. Grace, T. M. Dunn and D. L. Lubman, J. Phys. Chem., 1993, 97, 5820.

7 L. A. Philips and D. H. Levy, J. Chem. Phys., 1986, 85, 1327-1332.

8 G. Berden, W. L. Meerts and E. Jalviste, J. Chem. Phys., 1995, 103, 9596-9606.
9 C. Kang, T. M. Korter and D. W. Pratt, J. Chem. Phys., 2005, 122, 174301.

10 J. Zuclich, J. U. von Schütz and A. H. Maki, J. Am. Chem. Soc., 1974, 96, 710-714.

11 B. J. Fender, K. W. Short, D. K. Hahn and P. R. Callis, Int. J. Quantum Chem., 1999, 72, 347-356.

12 J. Küpper, D. W. Pratt, W. L. Meerts, C. Brand, J. Tatchen and M. Schmitt, Phys. Chem. Chem. Phys., 2010, 12, 4980-4988.

13 P. R. Callis, J. T. Vivian and L. S. Slater, Chem. Phys. Lett., 1995, 244, 53.

14 L. Serrano-Andrés and B. O. Roos, J. Am. Chem. Soc., 1996, 118, 185-195.

15 A. C. Borin and L. Serrano-Andrés, Chem. Phys., 2000, 262, 253-265.

16 L. Serrano-Andrés and A. C. Borin, Chem. Phys., 2000, 262, 267-283.

17 A. L. Sobolewski and W. Domcke, Chem. Phys. Lett., 1999, 315, 293-298.

18 C. Brand, J. Küpper, D. W. Pratt, W. L. Meerts, D. Krügler, J. Tatchen and M. Schmitt, Phys. Chem. Chem. Phys., 2010, 12, 4968-4997.

19 M. R. Hilaire, D. Mukherjee, T. Troxler and F. Gai, Chem. Phys. Lett., 2017, 685, 133-138.

20 M. R. Hilaire, I. A. Ahmed, C.-W. Lin, H. Jo, W. F. DeGrado and F. Gai, Proc. Natl. Acad. Sci. U. S. A., 2017, 114, 6005-6009.

21 B. N. Markiewicz, D. Mukherjee, T. Troxler and F. Gai, J. Phys. Chem. B, 2016, 210, 936-944.

22 O. Oeltermann, C. Brand, B. Engels, J. Tatchen and M. Schmitt, Phys. Chem. Chem. Phys., 2012, 14, 10266-10270.

23 C. Brand, B. Happe, O. Oeltermann, M. Wilke and M. Schmitt, J. Mol. Struct., 2013, 1044, 21-25.

24 B. Stuhlmann, A. Gräßle and M. Schmitt, Phys. Chem. Chem. Phys., 2014, 16, 899-905.

25 T. M. Korter, D. W. Pratt and J. Küpper, J. Phys. Chem. A, 1998, 102, 7211-7216.

26 A. Ahn, A. Min, C. J. Moon, J. H. Lee and M. Y. Choi, Chem. Phys. Lett., 2014, 616-617, 55-60.

27 S. Gerstenkorn and P. Luc, Atlas du spectre d'absorption de la molécule d'iode $14800-20000 \mathrm{~cm}^{-1}$, CNRS, Paris, 1986.

28 M. Schmitt, Habilitation, Heinrich-Heine-Universität, Math. Nat. Fakultät, Düsseldorf, 2000.

29 M. Schmitt, J. Küpper, D. Spangenberg and A. Westphal, Chem. Phys., 2000, 254, 349-361.

30 J. Wilke, M. Wilke, W. L. Meerts and M. Schmitt, J. Chem. Phys., 2016, 144, 044201.

31 K. Wohlfart, M. Schnell, J. U. Grabow and J. Küpper, J. Mol. Spectrosc., 2014, 247, 119-121.

32 R. Ahlrichs, M. Bär, M. Häser, H. Horn and C. Kölmel, Chem. Phys. Lett., 1989, 162, 165-169.

33 J. T. H. Dunning, J. Chem. Phys., 1989, 90, 1007-1023.

34 C. Hättig and F. Weigend, J. Chem. Phys., 2000, 113, 5154-5161.

35 C. Hättig and A. Köhn, J. Chem. Phys., 2002, 117, 6939-6951.

36 C. Hättig, J. Chem. Phys., 2002, 118, 7751-7761.

37 A. Hellweg, S. Grün and C. Hättig, Phys. Chem. Chem. Phys., 2008, 10, 1159-1169. 
38 P. Deglmann, F. Furche and R. Ahlrichs, Chem. Phys. Lett., 2002, 362, 511-518.

39 S. F. Boys and F. Bernardi, Mol. Phys., 1970, 19, 553-566.

40 P. J. Stephens, F. J. Devlin, C. F. Chabalowski and M. J. Frisch, J. Phys. Chem., 1994, 98, 11623-11627.

41 C. Lee, W. Yang and R. Parr, Phys. Rev. B: Condens. Matter Mater. Phys., 1988, 37, 785-789.

42 F. Furche and R. Ahlrichs, J. Chem. Phys., 2003, 117, 7433.

43 W. L. Meerts, M. Schmitt and G. Groenenboom, Can. J. Chem., 2004, 82, 804-819.

44 W. L. Meerts and M. Schmitt, Phys. Scr., 2005, 73, C47-C52.

45 W. L. Meerts and M. Schmitt, Int. Rev. Phys. Chem., 2006, 25, 353-406.

46 M. Schmitt and W. L. Meerts, Handbook of High Resolution Spectroscopy, John Wiley and Sons, 2011.

47 A. Ostenmeier, A. Gawelcyk and N. Hansen, Parallel Problem Solving from Nature, PPSN III, Springer, Berlin/Heidelberg, 1994.

48 N. Hansen and A. Ostermeier, Evol. Comput., 2001, 9, 159-195. 49 S. Grimme and M. Parac, ChemPhysChem, 2003, 4, 292-295.

50 M. Parac and S. Grimme, Chem. Phys., 2003, 292, 11-21.

51 S. Arulmozhiraja and M. L. Coote, J. Chem. Theory Comput., 2011, 8, 575-584.

52 M. Schmitt, R. Brause, C. Marian, S. Salzmann and W. L. Meerts, J. Chem. Phys., 2006, 125, 124309.

53 A. L. Sobolewski and W. Domcke, J. Phys. Chem. A, 2001, 105, 9275-9283.
54 A. L. Sobolewski, W. Domcke, C. Dedonder-Lardeux and C. Jouvet, Phys. Chem. Chem. Phys., 2002, 4, 1093-1100.

55 W. Domcke and A. L. Sobolewski, Science, 2003, 302, 1693-1694.

56 J. T. Hougen and J. K. G. Watson, Can. J. Phys., 1965, 43, 298-320.

57 A. Held, B. B. Champagne and D. W. Pratt, J. Chem. Phys., 1991, 95, 8732 .

58 M. Böhm, J. Tatchen, D. Krügler, K. Kleinermanns, M. G. D. Nix, T. A. LeGreve, T. S. Zwier and M. Schmitt, J. Phys. Chem. A, 2009, 113, 2456-2466.

59 G. A. Bickel, D. R. Demmer, E. A. Outhouse and S. C. Wallace, J. Chem. Phys., 1989, 91, 6013.

60 S. Arnold and M. Sulkes, J. Phys. Chem., 1992, 96, 4768.

61 J. Wilke, M. Wilke, C. Brand, W. L. Meerts and M. Schmitt, ChemPhysChem, 2016, 17, 2736-2743.

62 P. A. Steiner and W. Gordy, J. Mol. Struct., 1966, 21, 291-301.

63 H. S. Müller, A. Coutens, A. Walters, J.-U. Grabow and S. Schlemmer, J. Mol. Spectrosc., 2011, 267, 100-107.

64 G. Berden, W. L. Meerts, M. Schmitt and K. Kleinermanns, J. Chem. Phys., 1996, 104, 972-982.

65 C. Jacoby and M. Schmitt, Chem. Phys. Chem., 2004, 5, 1686-1694.

66 J. Kraitchman, Am. J. Phys., 1953, 21, 17.

67 W. L. Meerts, W. A. Majewski and W. M. van Herpen, Can. J. Phys., 1984, 62, 1293-1299. 